\title{
SUCCESSFUL TREATMENT OF TRAUMATIC ORBITAL APEX SYNDROME WITH LOW DOSE STEROIDS - A CASE REPORT
}

\section{Tharini Senthamizh* \\ Subashini Kaliaperumal}

ABSTRACT Traumatic Orbital Apex Syndrome is a rare complication of trauma presenting with visual loss, ophthalmoplegia, and anesthesia of cornea, forehead and maxillary regions. It requires immediate action as it poses great threat to permanent visual loss. The incidence of Traumatic orbital apex syndrome is very less compared to Superior Orbital fissure syndrome and traumatic optic neuropathy alone and only few cases have been reported till now. Management depends on the cause, those with displaced fracture fragments are treated by surgical decompression whereas those with edema or hematoma causing compression can be treated with steroids or surgical evacuation of hematoma. Previous reports have proved the usefulness of mega dose steroids in such cases.

We report a case of Traumatic Orbital Apex Syndrome who presented with painful proptosis, visual loss, ophthalmoplegia and loss of sensation in periorbital region. Imaging confirmed hematoma causing compression of neurovascular structures and hence a trial of low dose corticosteroids was started. Our patient showed dramatic improvement in signs and symptoms with complete recovery in three weeks. Low dose steroids can be considered as an alternative to mega dose steroids to treat patients with indirect traumatic Orbital Apex Syndrome, thereby reducing the necessity of surgical evacuation in such cases.

KEYWORDS : Trauma, Traumatic Orbital apex syndrome, proptosis, hematoma, low dose steroids

\section{INTRODUCTION}

Orbital apex syndrome (OAS), also known as Jacod syndrome, is defined as simultaneous dysfunction of the optic nerve and cranial nerves manifesting with vision loss, ptosis and complete internal and external ophthalmoplegia, as a result of processes occurring in the region of optic canal and the superior orbital fissure. The etiology includes vascular, infectious, inflammatory, neoplastic, iatrogenic and rarely traumatic. OAS has been observed to occur in both blunt and penetrating trauma. ${ }^{1}$ Onset of symptoms following trauma is immediate, although delayed onset after 72 hours has been reported. ${ }^{2}$ Treatment of traumatic OAS depends on the type of injury. OAS due to impingement of bony fragments following cranio-maxillary trauma is treated by immediate surgical intervention whereas compression due to hemorrhage or edema can be treated by corticosteroids. There is no clear consensus regarding the medical management of OAS and dose of steroids.We report a case of OAS who presented with proptosis, external ophthalmoplegia and loss of forehead and orbital sensation with visual loss following road traffic accident. As there was no evidence of bony compression, we treated the patient with low dose steroids and the patient had complete recovery of vision and ocular motility within three weeks.

\section{CASE REPORT}

Al3-year-old boy allegedly had a road traffic accident two days back, while he was riding in the pillion, the two-wheeler accidently hit the tree by the roadside. He developed loss of consciousness and swelling of the left eyelid and hence was brought to the emergency department. Ophthalmic examination of both the eyes done on day one was normal. Next day, when he regained consciousness, he noticed protrusion of left eye associated with pain, loss of vision and inability to move in any direction. He had no history of fever, vomiting or nasal bleeding. On day two, repeat ophthalmic examination of his left eye revealed tender eyeball with abaxial proptosis with a deviation of $8 \mathrm{~mm}$ downwards and $10 \mathrm{~mm}$ outwards (fig- 1 ).

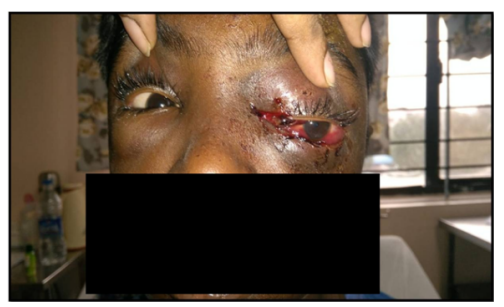

Fig l: Left eye showing Ābaxial Proptosis with hemorrhagic chemosis

There was no increase in protrusion of eyeball following Valsalva maneuver or dependent position. Also, there was no pulsation or thrill over the proptosis. There was a total restriction of movements in all gazes and loss of sensation in corneal, supraorbital and maxillary regions. He could not perceive any light stimulus in left eye. There was edema of both upper and lower eyelids and hemorrhagic chemosis of the conjunctiva. Pupillary examination in left eye revealed a dilatation of $4 \mathrm{~mm}$ with Relative Afferent Pupillary defect on swinging flashlight test. Intraocular pressure (IOP) was $32 \mathrm{mmHg}$ in left eye. Fundus examination of left eye was normal except for macular folds. Right eye examination was normal. Systemic examination was normal. An emergency Computed Tomography scan (CT) showed depressed fracture of roof of orbit and fracture of medial wall. Intra orbital extraconal swelling was noted in supero-medial aspect with narrowing of optic canal and superior orbital fissure (fig2).This was confirmed in MRI (Magnetic Resonance Imaging) which revealed compression of orbital apex due to edema around the apex with hematoma noted in supero-medial orbit.

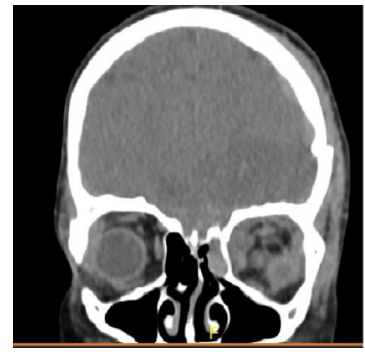

Fig 2: CT orbits(coronal section) showing hematoma in the 
supero-medial aspect of left orbit with edema around the optic nerve

Patient underwent lateral canthotomy and cantholysis on the same day following which the vision remained the same. Hence the patient was started on intravenous methylprednisolone $\mathrm{lgm} /$ day. There was a dramatic improvement of vision in left eye to $6 / 24$ on day four and to 6/12 on day eight of steroid treatment. Extra ocular movements improved with minimal restriction in all gazes on day eight. On day fourteen, the patient improved completely with $6 / 6$ vision in left eye and normal extra ocular movements with 15 degrees exotropia (fig 3).Pupil examination showed brisk response to both direct and consensual light reflex and the corneal and periorbital sensations also improved. IOP was $14 \mathrm{mmHg}$. Final follow up at three weeks revealed normal vision with full extra ocular movements and resolution of proptosis and a normal MRI (Fig 4).

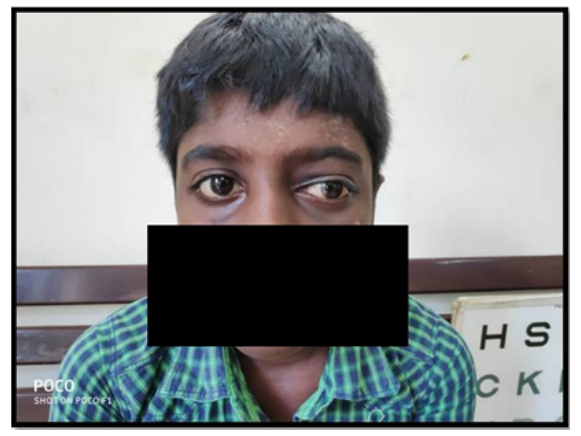

Fig 3: Improvement in Proptosis with mild exotropia noted on day 14 following steroid therapy

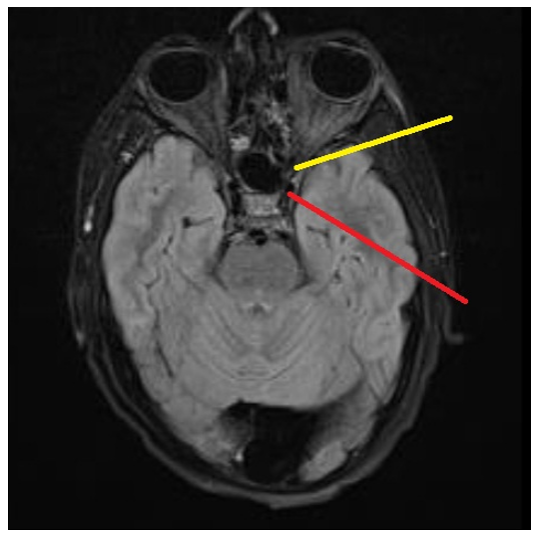

Fig 4: Axial TlWeighted MRI through orbits on day 21 demonstrate normal appearing optic canal (shown in yellow arrow) and superior orbital fissure (shown in red arrow)

\section{DISCUSSION}

Orbital apex is a closed space formed by Superior orbital fissure and Optic foramen. Important neuro-vascular structures pass through this area which makes it vulnerable to profound functional loss even to smaller pathologies. ${ }^{3} \mathrm{Clinical}$ features of OAS includes visual loss due to Optic neuropathy, ophthalmoplegia resulting from Oculomotor, Trochlear and Abducent nerve palsies and anesthesia in the region supplied by ophthalmic division of Trigeminal nerve. Patients with traumatic OAS usually present with coma and hence the symptoms are usually neglected till the patient regains consciousness. This leads to delay in diagnosis and treatment. Hence, it is mandatory to do an ophthalmic examination once the patient regains consciousness. ${ }^{4}$ Our patient also had loss of consciousness for one day and he noticed loss of vision only the next day. Although we did an ophthalmic examination on the day of presentation, there was no RAPD or proptosis and visual acuity examination was not possible as the patient was in coma. The very next day, when the patient became conscious, ophthalmic examination was done which revealed severe proptosis and other signs of OAS.This delay in presentation have to alert the clinicians to take immediate action so as to prevent permanent visual loss.

Owing to the rarity of traumatic OAS, there is no well established treatment protocol for this condition. Direct injury to the orbital apex by displaced fracture fragments has to be managed by surgical decompression of the optic nerve. Radiologically or clinically evident impingement should be treated immediately by surgical intervention to avoid irreversible ischemic damage to the optic nerve. ${ }^{5}$ Several reports have proven success in treating direct $\mathrm{OAS}$ with surgical decompression either by transcranial $^{6}$ or trans-nasal endoscopic approach. ${ }^{4}$ On the other hand, indirect injury to orbital apex can be caused by retro orbital hematoma or edema. Steroid therapy has been found beneficial in few previously reported cases of traumatic superior orbital fissure syndrome (SOFS). Chen et al reported that those patients who had steroid therapy had better neurological recovery than those with observation alone $(70 \%$ vs. $42.1 \%)$. ${ }^{7}$ Postma et al reported complete recovery within 3 months with short term use of dexamethasone $(4 \mathrm{mg}$ every 6 hours) in a patient who presented with traumatic SOFS associated with sphenoid and zygomatico-maxillary fracture. In a study by Acarturk et al megadose steroid $(30 \mathrm{mg} / \mathrm{kg}$ intravenous methyl prednisolone loading dose followed by $5.4 \mathrm{mg} / \mathrm{kg}$ per hour for 48 hours) was found to be beneficial. ${ }^{9} \mathrm{To}$ our knowledge, our case report is the first to use low dose steroids (Intravenous methylprednisolone lgm/day for 3 days followed by oral steroids $\mathrm{lmg} / \mathrm{kg} /$ day for 11 days ) to treat traumatic orbital apex syndrome with rapid improvement to near normal, within two weeks. We used low dose steroids to avoid the side effects of mega dose steroids and to reduce the risk of infection in such patients.

Our patient had hematoma compressing the optic nerve without bony impingement. As we visualized a dramatic improvement by using low dose steroids, surgical evacuation was not needed in our patient. Study by Bracken et al has proved detrimental effects of megadose steroids in patients with spinal cord injury which should be taken into consideration before opting for such high doses of steroids in such cases where there can be associated intracranial injuries. ${ }^{10}$

In conclusion, awareness about traumatic OAS, is needed among physicians handling trauma cases, for prompt referral of such cases to Ophthalmologists to prevent irreversible visual loss. As delay in presentation is common in such cases, it is mandatory do an ophthalmic examination in all head injury patients once they recover from coma. Low dose steroids can be considered as an effective alternative in patients with traumatic OAS but this has to be proven by prospective clinical trials.

\section{REFERENCES}

1. Anderson RL, Panje WR, Gross CE: Optic nerve blindness following blunt forehead trauma. Ophthalmology 1982; 89:445-55.

2. Jarrahy R, Cha ST, Shahinian HK: Retained foreign body in the orbit and cavernous sinus with delayed presentation of superior orbital fissure syndrome: case report.J Craniofac Surg 2001; 12:82-86.

3. Peter NM, Pearson AR. Orbital apex syndrome from blunt ocular trauma. Orbit 2010(Feb); 29(1):42-4.

4. Tong Y, Chen G, Jiang F, Wu W. Successful delayed treatment of traumatic orbital apex syndrome by nasal endoscopic decompression surgery. Indian J Ophthalmol 2015; 63:728-30.

5. Imaizumi A, Ishida K, Ishikawa, Y, Nakayoshi, I. Successful treatment of the traumatic orbital apex syndrome due to direct bone compression. Craniomaxillofac Trauma Reconstr 2014; 7(04):318-22.

6. Shokri T, Zacharia BE, Lighthall JG.Traumatic Orbital apex Syndrome: An Uncommon Sequela of facial trauma. Ear Nose Throat J 2019; 98(10):609-12.

7. Chen CT, Chen YR. Traumatic superior orbital fissure syndrome: Current management. Craniomaxillofac Trauma Reconstr 2010; 3:9-16.

8. Postma M P, Seldomridge G W, Vines F S.Superior Orbital fissure syndrome and bilateral internal carotid pseudoaneurysms. J Oral Maxillofac surg1990; 48:503-8.

9. Acarturk S, Sekucoglu T, Kesiktas E. Mega dose corticosteroid treatment for traumatic superior orbital fissure and orbital apex syndromes. Ann Plast Surg 2004; 53:60-64

10. Bracken MB, Collins WF, Freeman DF, Shepard MJ,Wagner FW, Silten RM, et al. Efficacy of methylprednisolone in acute spinal cord injury. JAMA 1984; 251:45-52. 